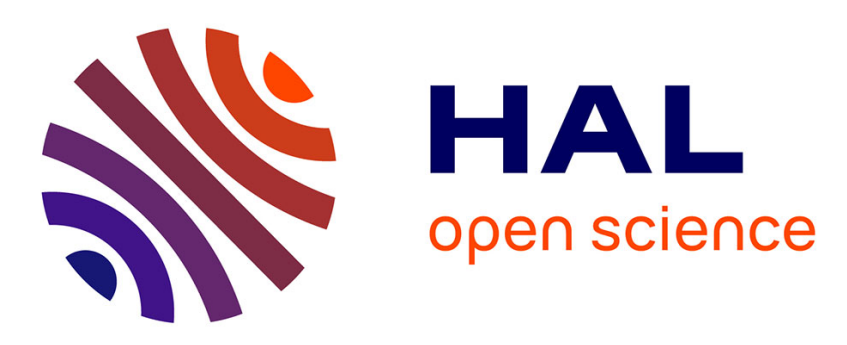

\title{
A New Normalized Supervised Edge Detection Evaluation
}

\author{
Hasan Abdulrahman, Baptiste Magnier, Philippe Montesinos
}

\section{To cite this version:}

Hasan Abdulrahman, Baptiste Magnier, Philippe Montesinos. A New Normalized Supervised Edge Detection Evaluation. Pattern Recognition and Image Analysis 8th Iberian Conference, IbPRIA 2017, Faro, Portugal, June 20-23, 2017, Proceedings, pp.203-213, 2017, Lecture Notes in Computer Science (including subseries Lecture Notes in Artificial Intelligence and Lecture Notes in Bioinformatics), 978-3-319-58837-7. 10.1007/978-3-319-58838-4_23. hal-01940333

\section{HAL Id: hal-01940333 https://hal.science/hal-01940333}

Submitted on 30 Nov 2018

HAL is a multi-disciplinary open access archive for the deposit and dissemination of scientific research documents, whether they are published or not. The documents may come from teaching and research institutions in France or abroad, or from public or private research centers.
L'archive ouverte pluridisciplinaire HAL, est destinée au dépôt et à la diffusion de documents scientifiques de niveau recherche, publiés ou non, émanant des établissements d'enseignement et de recherche français ou étrangers, des laboratoires publics ou privés. 


\title{
A NEW NORMALIZED SUPERVISED EDGE DETECTION EVALUATION
}

\author{
Hasan Abdulrahman, Baptiste Magnier, and Philippe Montesinos \\ Ecole des Mines d'Alès, Parc scientifique Georges Besse, 30000 Nîmes, France
}

\begin{abstract}
In digital images, edges characterize object boundaries, then their detection remains a crucial stage in numerous applications. To achieve this task, many edge detectors have been designed, producing different results, with different qualities. Evaluating the response obtained by these detectors has become a crucial task. In this paper, several referenced-based boundary detection evaluations are detailed, pointing their advantages and disadvantages through concrete examples of edge images. Then, a new supervised edge map quality measure is proposed, comparing a ground truth contour image, the candidate contour image and their associated spacial nearness. Compared to other boundary detection assessments, this new method has the advantage to be normalized and remains a more reliable edge map quality measure.
\end{abstract}

Keywords: Edge detection, distance measure, supervised evaluation.

\section{IMPORTANCE OF A NEW ERROR MEASURE}

In image processing tasks, edge detection remains a key point in many applications. Boundaries include the most important structures of the image, and an efficient boundary detection method should create a contour image containing edges at their correct locations with a minimal of misclassified pixels. Different algorithms have been developed in the past, but few of them give an objective performance comparison. The evaluation process should produce a result that correlates with the perceived quality of the edge image, which is relied on human judgment. In other words, a reliable edge map should characterize all the relevant structures of an image. On the other hand, a minimum of spurious pixels or holes (oversights) must be created by the edge detector at the same time. Therefore, an efficient evaluation can be used to assess and improve an algorithm, or to optimize edge detector parameters [1].

The measurement process can be classified into either an unsupervised or a supervised evaluation criteria. The first class of methods exploits only the input contour image and gives a score of coherence that qualifies the result given by the algorithm [1]. The second one computes a dissimilarity measure between a segmentation result and a ground truth obtained from synthetic data or an expert judgment (i.e. manual segmentation) [2][3][4]. This work focusses on comparisons of supervised assessment of edge detection evaluations. Furthermore, a new supervised edge map quality measure based on the distances of misplaced pixels is presented and compared to the others, using synthetic and real images. 


\section{SUPERVISED IMAGE CONTOUR EVALUATIONS}

As introduced above, a supervised evaluation process estimates scores between a ground truth and a candidate edge map. In image processing, the Structural Similarity Index $(S S I M)$ corresponds to an image quality evaluation, which estimates the visual impact of gray scale shifts in an image [5]. Otherwise, contours (binary images) could be evaluated counting the number of erroneous pixels, but also throughout spatial distances of misplaced or oversights contours.

\subsection{Error measures involving only the confusion matrix}

Let $G_{t}$ be the reference contour map corresponding to ground truth and $D_{c}$ the detected contour map of an image $I$. Comparing pixel per pixel $G_{t}$ and $D_{c}$, common positive or negative presence of points is the first criterion to be assessed. A basic evaluation is compounded of statistics issued of a confusion matrix. To that effect, $G_{t}$ and $D_{c}$ are combined. Afterward, denoting $|\cdot|$ the cardinality of a set, all points are partitioned into four sets:

- True Positive points (TPs), common points of $G_{t}$ and $D_{c}: T P=\left|D_{c} \cap G_{t}\right|$,

- False Positive points (FPs), spurious detected edges of $D_{c}: F P=\left|D_{c} \cap \neg G_{t}\right|$,

- False Negative points (FNs), missing boundary points of $D_{c}: F N=\left|\neg D_{c} \cap G_{t}\right|$, - True Negative points (TNs), common non-edge points: $T N=\left|\neg D_{c} \cap \neg G_{t}\right|$.

In one hand, let us consider boundary detection of images, FPs appear in the presence of noise, texture or other contours influencing the filter used by the edge detection operator. In the other hand, FNs represent holes in a contour of $D_{c}$. Finally, a wrong threshold of the segmentation could generate both FPs and FNs. Computing only FPs and FNs enables a segmentation assessment [6][7], and a reliable edge detection should minimize the following indicators [3]:

$$
\left\{\begin{array}{c}
\text { Over-detection error : } \operatorname{Over}\left(G_{t}, D_{c}\right)=\frac{F P}{|I|+\left|G_{t}\right|}, \\
\text { Under-detection error : } \operatorname{Under}\left(G_{t}, D_{c}\right)=\frac{F N}{\left|G_{t}\right|}, \\
\text { Localization-error : } \operatorname{Loc}\left(G_{t}, D_{c}\right)=\frac{F P+F N}{|I|}
\end{array}\right.
$$

Additionally, the Performance measure $P_{m}^{*}$ presented in Table 1 considers directly at the same time the three entities TP, FP and $F N$ to assess an a binary image. The obtained score reflects the percentage of statistical errors.

Another way to display evaluations are Receiver Operating Characteristic (ROC) [8] curves or Precision-Recall (PR) [9], involving True Positive Rates $\left(T P R=\frac{T P}{T P+F N}\right)$ and False Positive Rates $\left(F P R=\frac{F P}{F P+T N}\right)$. Derived from $T P R$ and FPR, the three measures $\Phi, \chi^{2}$ and $F_{\alpha}$ (see Table 1 ) are frequently used in edge detection assessment.

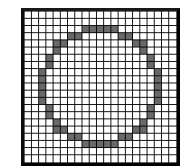

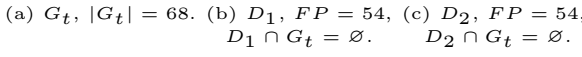

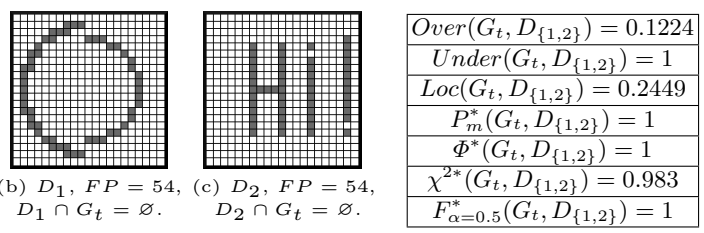

\begin{tabular}{|c|}
\hline $\operatorname{FoM}\left(G_{t}, D_{1}\right)=0.22$ \\
\hline $\operatorname{FoM}\left(G_{t}, D_{2}\right)=0.60$ \\
\hline \hline$H\left(G_{t}, D_{1}\right)=1.41$ \\
\hline$H\left(G_{t}, D_{2}\right)=7.67$ \\
\hline \hline$\Delta_{w}^{k}\left(G_{t}, D_{1}\right)=0.96$ \\
\hline$\Delta_{w}^{k}\left(G_{t}, D_{2}\right)=2.31$ \\
\hline
\end{tabular}

Fig. 1. Evaluations issued of a confusion matrix can be the same for different $D_{c}$. For the two candidate edge images, number of FPs and number of FNs are the same. 
Table 1. List of error measures, $k=1$ or $k=2$ are the most common values.

\begin{tabular}{|c|c|c|}
\hline Error measure name & Formulation & Parameters \\
\hline $\begin{array}{l}\text { Performance } \\
\text { measure }[11]\end{array}$ & $P_{m}^{*}\left(G_{t}, D_{c}\right)=1-\frac{T P}{T P+F P+F N}$ & None \\
\hline $\begin{array}{l}\text { Complemented } \Phi \text { mea- } \\
\text { sure [12] }\end{array}$ & $\Phi^{*}\left(G_{t}, D_{c}\right)=1-\frac{T P R \cdot T N}{T N+F P}$ & None \\
\hline $\begin{array}{l}\text { Complemented } \quad \chi^{2} \\
\text { measure }[13]\end{array}$ & $\chi^{2 *}\left(G_{t}, D_{c}\right)=1-\frac{T P R-T P-F P}{1-T P-F P} \cdot \frac{T P+F P+F P R}{T P+F P}$ & None \\
\hline $\begin{array}{l}\text { Complemented } \quad F_{\alpha} \\
\text { measure }[9]\end{array}$ & $F_{\alpha}^{*}\left(G_{t}, D_{c}\right)=1-\frac{P R E C \cdot T P R}{\alpha \cdot T P R+(1-\alpha) \cdot P R E C}, \quad$ with $P R E C=\frac{T P}{T P+F P}$ & $\alpha \in] 0 ; 1]$ \\
\hline Pratt's FoM [14] & $\operatorname{FoM}\left(G_{t}, D_{c}\right)=1-\frac{1}{\max \left(\left|G_{t}\right|,\left|D_{c}\right|\right)} \cdot \sum_{p \in D_{c}} \frac{1}{1+\kappa \cdot d_{G_{t}}^{2}(p)}$ & $\kappa \in] 0 ; 1]$ \\
\hline FoM revisited $[15]$ & $F\left(G_{t}, D_{c}\right)=1-\frac{1}{\left|G_{t}\right|+\beta \cdot F P} \cdot \sum_{p \in G_{t}} \frac{1}{1+\kappa \cdot d_{D_{c}}^{2}(p)}$ & $\begin{array}{l}\kappa \in] 0 ; 1] \text { and } \\
\beta \in \mathbb{R}^{+}\end{array}$ \\
\hline $\begin{array}{l}\text { Combination of FoM } \\
\text { and statistics }[16]\end{array}$ & $d_{4}\left(G_{t}, D_{c}\right)=\frac{1}{2} \cdot \sqrt{\frac{\left(T P-\max \left(\left|G_{t}\right|,\left|D_{c}\right|\right)\right)^{2}+F N^{2}+F P^{2}}{\left(\max \left(\left|G_{t}\right|,\left|D_{c}\right|\right)\right)^{2}}+F o M\left(G_{t}, D_{c}\right)}$ & $\begin{array}{l}\kappa \in] 0 ; 1] \text { and } \\
\beta \in \mathbb{R}^{+}\end{array}$ \\
\hline Yasnoff measure [17] & $\Upsilon\left(G_{t}, D_{c}\right)=\frac{100}{|I|} \cdot \sqrt{\sum_{p \in D_{c}} d_{G_{t}}^{2}(p)}$ & None \\
\hline Hausdorff distance [18] & $H\left(G_{t}, D_{c}\right)=\max \left(\max _{p \in D_{c}}\left(d_{G_{t}}(p)\right), \max _{p \in G_{t}}\left(d_{D_{c}}(p)\right)\right)$ & None \\
\hline Maximum distance $[2]$ & $f_{2} d_{6}\left(G_{t}, D_{c}\right)=\max \left(\frac{1}{\left|D_{c}\right|} \cdot \sum_{p \in D_{c}} d_{G_{t}}(p), \frac{1}{\left|G_{t}\right|} \cdot \sum_{p \in G_{t}} d_{D_{c}}(p)\right)$ & None \\
\hline Distance to $G_{t}[19][4]$ & $D^{k}\left(G_{t}, D_{c}\right)=\frac{1}{\left|D_{c}\right|} \cdot \sqrt[k]{\sum_{p \in D_{c}} d_{G_{t}}^{k}(p)}, \quad k=1$ for [19] & $k \in \mathbb{R}^{+}$ \\
\hline $\begin{array}{l}\text { Oversegmentation } \\
{[20][21]}\end{array}$ & $\Theta\left(G_{t}, D_{c}\right)=\frac{1}{F P} \cdot \sum_{p \in D_{c}}\left(\frac{d_{G_{t}}(p)}{\delta_{T H}}\right)^{k}, \quad k=\delta_{T H}=1$ for $[20]$ & $\begin{array}{l}\text { for }[21]: k \in \mathbb{R}^{+} \\
\text {and } \delta_{T H} \in \mathbb{R}_{*}^{+}\end{array}$ \\
\hline $\begin{array}{l}\text { Undersegmentation } \\
{[20][21]}\end{array}$ & $\Omega\left(G_{t}, D_{c}\right)=\frac{1}{F N} \cdot \sum_{p \in G_{t}}\left(\frac{d_{D_{c}}(p)}{\delta_{T H}}\right)^{k}, \quad k=\delta_{T H}=1$ for $[20]$ & $\begin{array}{l}\text { for }[21]: k \in \mathbb{R}^{+} \\
\text {and } \delta_{T H} \in \mathbb{R}_{*}^{+}\end{array}$ \\
\hline $\begin{array}{l}\text { Symmetric distance } \\
{[2][4]}\end{array}$ & $S^{k}\left(G_{t}, D_{c}\right)=\sqrt[k]{\frac{\left.\sum_{p \in D_{c}} d_{G_{t}}^{k}(p)\right)+\sum_{p \in G_{t}} d_{D_{c}}^{k}(p)}{\left|D_{c} \cup G_{t}\right|}}$ & $k \in \mathbb{R}^{+}$ \\
\hline $\begin{array}{l}\text { Baddeley's Delta Met- } \\
\text { ric }[22]\end{array}$ & $\Delta^{k}\left(G_{t}, D_{c}\right)=\sqrt[k]{\frac{1}{|I|} \cdot \sum_{p \in I}\left|w\left(d_{G_{t}}(p)\right)-w\left(d_{D_{c}}(p)\right)\right|^{k}}$ & $\begin{array}{l}k \in \mathbb{R}^{+} \text {and a } \\
\text { convex function } \\
w: \mathbb{R} \mapsto \mathbb{R}\end{array}$ \\
\hline $\begin{array}{l}\text { Edge map quality } \\
\text { measure }[23]\end{array}$ & $D_{p}\left(G_{t}, D_{c}\right)=\frac{1 / 2}{|I|-\left|G_{t}\right|} \cdot \sum_{p \in D_{c}}\left(1-\frac{1}{1+\alpha \cdot d_{G_{t}}^{2}(p)}\right)+\frac{1 / 2}{\left|G_{t}\right|} \cdot \sum_{p \in G_{t}}\left(1-\frac{1}{1+\alpha \cdot d_{G_{t} \cap D_{c}}^{2}(p)}\right)$ & $\alpha \in] 0 ; 1]$ \\
\hline $\begin{array}{l}\text { Magnier et al. measure } \\
{[24]}\end{array}$ & $\Gamma\left(G_{t}, D_{c}\right)=\frac{F P+F N}{\left|G_{t}\right|^{2}} \cdot \sqrt{\sum_{p \in D_{c}} d_{G_{t}}^{2}(p)}$ & None \\
\hline
\end{tabular}

$P_{m}^{*}, \Phi, \chi^{2}$ and $F_{\alpha}$ measures are normalized and decrease with the quality of the detection; a score equal to 0 qualifies a perfect segmentation. These measures evaluate the comparison of two edge images, pixel per pixel, tending to penalize severely a misplaced contour (even weak). So they do not indicate significant variations of the desired contour shapes through an evaluation (as illustrated in Fig. 1). As this penalization tends to be too severe, some evaluations issued from the confusion matrix recommend a spatial tolerance, particularly for assimilation of TPs [8] [9]. This inclusion could be carried by a distance threshold or a dilation of $D_{c}$ and/or $G_{t}$. A such strategy of assimilation leads to counting several near contours as parallel stripes to the desired boundary. Tolerating a distance from the true contour and integrating several TPs for one detected contour are opposite to the principle of unicity in edge detection expressed by the 3rd Canny criteria: an optimal edge detector must produce a single response for one contour [10]. Finally, to perform an edge evaluation, the assessment should penalize a misplaced edge point proportionally to the distance to its true location. 


\subsection{Assessment involving distances of misplaced pixels}

Existing quality measures involving distances: A reference-based edge map quality measure requires that a displaced edge expects to be penalized in function not only of the FPs and/or FNs but also in function of the distance to the position they should be located at. Table 1 reviews the most relevant existing measures. The common feature between these evaluators corresponds to the error distance $d_{G_{t}}(p)$ or $d_{D_{c}}(p)$. Indeed, for a pixel $p \in D_{c}, d_{G_{t}}(p)$ represents the minimal distance between $p$ and $G_{t}$, whereas if $p \in G_{t}, d_{D_{c}}(p)$ corresponds to the minimal distance between $p$ and $D_{c}$. This distance refers to the Euclidean distance, even though some authors involve others, see [4]. Thus, a measure computing an error distance only in function of $d_{G_{t}}$ estimates the divergence of FPs, which corresponds to an over-segmentation (cases $\Upsilon, D^{k}, \Theta, F_{O} M$ and $\Gamma$ ). On the contrary, the sole use of a distance $d_{D_{c}}$ enables an estimation of the FNs divergence, representing an under-segmentation, as $\Omega$ distance measure. A measure widely computed in matching techniques is represented by the Hausdorff distance $H$ which estimates the mismatch of two sets of points [18]. This max-min distance could be strongly deviated by only one pixel which can be positioned sufficiently far from the pattern (illustrated in Fig. 2); so the measured distance becomes that between the pattern and the (erroneous) point, disturbing in that case the score of $H$. To improve $H$ such that it becomes less sensitive to outliers, an idea is to compute $H$ with a proportion of the maximum distances (for example $5 \%$ of the values [18]); let us note $H_{n \%}$ this measurement for $n \%$ of values $\left(n \in \mathbb{R}_{*}^{+}\right)$. One of the most popular descriptor corresponds to Figure of Merit $(F o M)$. This distance measure ranges from 0 to 1 , where 0 corresponds to a perfect segmentation [14], but computes only distances of the FPs [22]. Thus, some improvements have been developed as $F$ and $d_{4}$. Furthermore, as concluded in [3], a complete and optimum edge detection evaluation measure should combine assessments of both over- and under-segmentation, as in $S^{k}, \Delta_{w}^{k}$ and $D_{p}$. As an example, inspired by $f_{2} d_{6}$ [2], another way is to consider the combination of both FoM $\left(G_{t}, D_{c}\right)$ and FoM $\left(D_{c}, G_{t}\right)$, as the two following formulas:

$$
\begin{gathered}
\text { Symmetric FoM: } \operatorname{SFoM}\left(G_{t}, D_{c}\right)=\frac{1}{2} \cdot \operatorname{FoM}\left(G_{t}, D_{c}\right)+\frac{1}{2} \cdot \operatorname{FoM}\left(D_{c}, G_{t}\right) \\
\text { Maximum FoM: } \operatorname{MFoM}\left(G_{t}, D_{c}\right)=\max \left(\operatorname{FoM}\left(G_{t}, D_{c}\right), \operatorname{FoM}\left(D_{c}, G_{t}\right)\right) .
\end{gathered}
$$

Finally, $S F o M$ and $M F o M$ take into account both distances of FNs (i.e. $d_{D_{c}}$ ) and FPs (i.e. $d_{G_{t}}$ ), so they can compute a global evaluation of a contour image.

Another way to compute a global measure is presented in [23] with the normalized edge map quality measure $D_{p}$. In fact, this distance measure seems

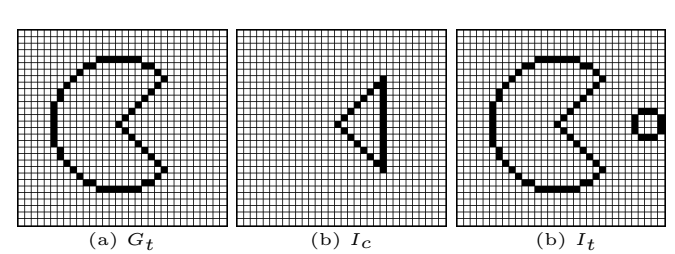

\begin{tabular}{|c|c|c|}
\hline Measure & $I_{t}$ & $I_{c}$ \\
\hline FoM & 0.63 & 0.17 \\
\hline$F$ & 0.72 & 0.18 \\
\hline$d_{4}$ & 0.62 & 0.15 \\
\hline SFoM & 0.64 & 0.17 \\
\hline MFoM & 0.82 & 0.58 \\
\hline$D_{P}$ & 0.33 & 0.007 \\
\hline$\Upsilon$ & 1.14 & 3.30 \\
\hline$H$ & 109 & 130 \\
\hline$H_{5 \%}$ & 10.39 & 11.01 \\
\hline
\end{tabular}

\begin{tabular}{|c|c|c|}
\hline Measure & $I_{t}$ & $I_{c}$ \\
\hline$D_{k=2}^{k}$ & 0.39 & 0.47 \\
\hline$\theta_{\delta_{T H}=1}$ & 2.74 & 9.07 \\
\hline$\theta_{\delta_{T H}=5}$ & 0.37 & 3.35 \\
\hline$\Omega_{\delta_{T H}=1}$ & 7.91 & 0 \\
\hline$\Delta^{k}$ & 6.05 & 1.20 \\
\hline$f 2 d 6$ & 5.79 & 1.60 \\
\hline$S_{k=2}^{k}$ & 5.97 & 2.84 \\
\hline$\Gamma$ & 0.19 & 0.12 \\
\hline$\Psi$ & 0.94 & 0.12 \\
\hline
\end{tabular}

Fig. 2. The scores of the over-segmentation evaluations are higher for $I_{t}$ whereas $I_{t}$ is more closer visually to $G_{t}$ than $I_{c}$. 
similar to $S F o M$ with different coefficients. However, both the left and the right terms are composed of a $\frac{1}{2}$ coefficient, so in the presence of only a pure underor over-segmentation, the score of $D_{p}$ does not attain over $\frac{1}{2}$.

A new edge detection assessment measure: In [24] is developed a normalized measure of the edge detection assessment, denoted $\Gamma$. This function represents an over-segmentation measure which depends also of $F N$ and $F P$. As this measure is not sufficiently efficient concerning FNs because it does not consider $d_{D_{c}}$ for false negative points (see Fig. 7). Thus, inspired by $S^{k}$, the new measure $\Psi$ holds different coefficients changing the behavior of the measure:

$$
\Psi\left(G_{t}, D_{c}\right)=\frac{F P+F N}{\left|G_{t}\right|^{2}} \cdot \sqrt{\sum_{p \in G_{t}} d_{D_{c}}^{2}(p)+\sum_{p \in D_{c}} d_{G_{t}}^{2}(p)}
$$

Compared to $\Gamma, \Psi$ improves the measurement by combining both $d_{G_{t}}$ and $d_{D_{c}}$ (illustrated in Fig. 7). Authors of $\Gamma$ have studied the influence of the coefficient in different concrete cases [24]. They concluded that such a formulation must take into consideration all observable cases and theoretically observable. In fact, a performing measure has to take into account all the following input parameters $\left|G_{t}\right|, F N$ and $F P$ whereas the image dimensions should not be considered. Thus, the parameter $\frac{F P+F N}{\left|G_{t}\right|^{2}}$ seems a good compromise and has been introduced to the new formula of assessment $\Psi$.

\subsection{Normalization of the edge detection evaluation:}

In order to compare each boundary detection assessments, all measures must be normalized, but also indicate the same information: an error measure close to 1 means a poor segmentation whereas a value close to 0 indicates a good segmentation. Thereby, the values of $F o M, F, d_{4}$ and $D_{p}$ belongs to $[0,1]$. However, concerning other distance measures of table 1 , a normalization is required. Introduced in [24], a formula called Key Performance Indicator $(K P I)$ gives value close to 1 for a poor segmentation; alternatively, a KPI value close to 0 translates a good segmentation:

$$
\begin{aligned}
K P I_{u}:[0 ; \infty[ & \mapsto[0 ; 1[ \\
u & \rightarrow 1-\frac{1}{1+u^{h}} .
\end{aligned}
$$

where the parameter $u$ represents a distance error and $h$ a constant $\left(h \in \mathbb{R}_{*}^{+}\right)$.

An undeniable parameter of $K P I$ formula is the power of the denominator term called $h$. Inasmuch as KPI depends on its value, it evolves more or less quickly around 0.5 and embodies a range of observable cases. The advice to choose values between 1 and 2 can be easily checked. Otherwise, the more KPI evolution will be abrupt, the less the transition between 0.5 and 1 will be marked. As a compromise, fixing the power at the golden ratio $\phi \simeq 1.6180339887$, the measurement becomes not too strong in the presence a small measure score, but increases to 1 for a high score of the distance measure, see [24].

\section{EXPERIMENTAL RESULTS}

Experiments realized in this part aim to be the most accomplished, thus the more close and realistic of the reality. In respect of these directives, in a first 

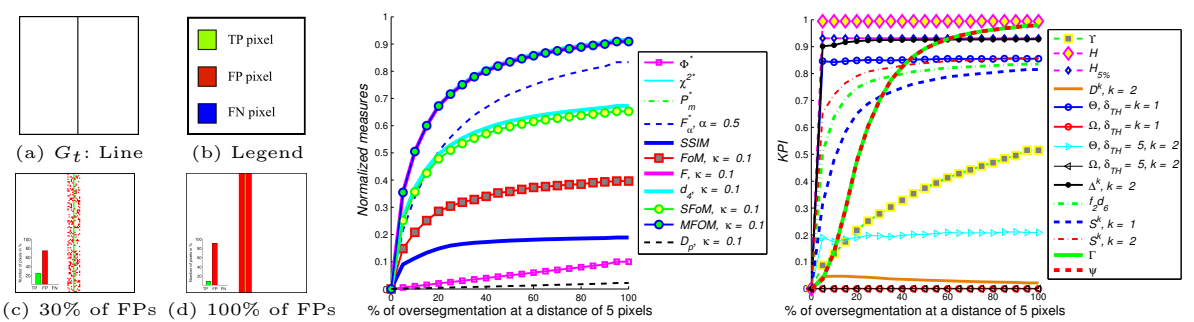

Fig. 3. Measures scores in function of the over-segmentation in the contour area.
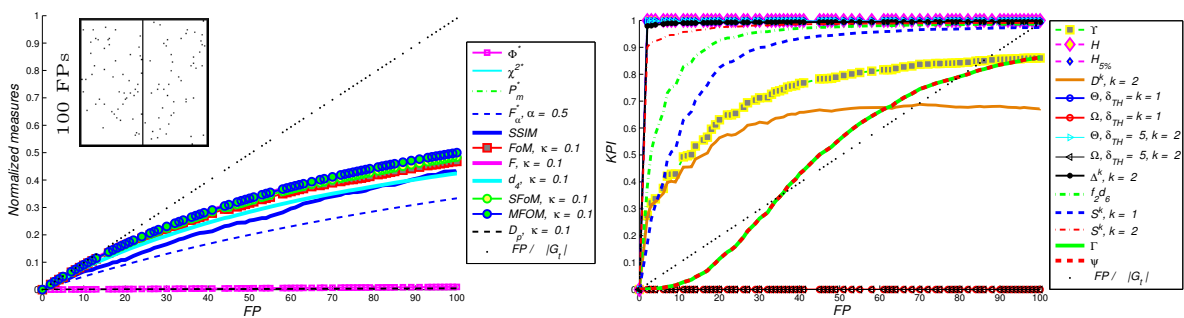

Fig. 4. Evolution of the dissimilarity measures in function of the FPs addition.
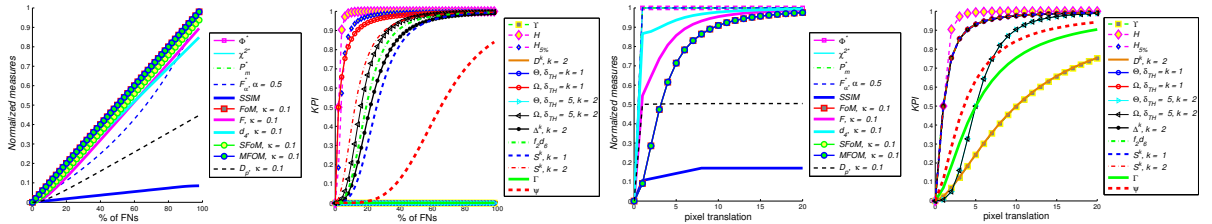

Fig. 5. Measure scores in function of the FNs addition and the edge translation.
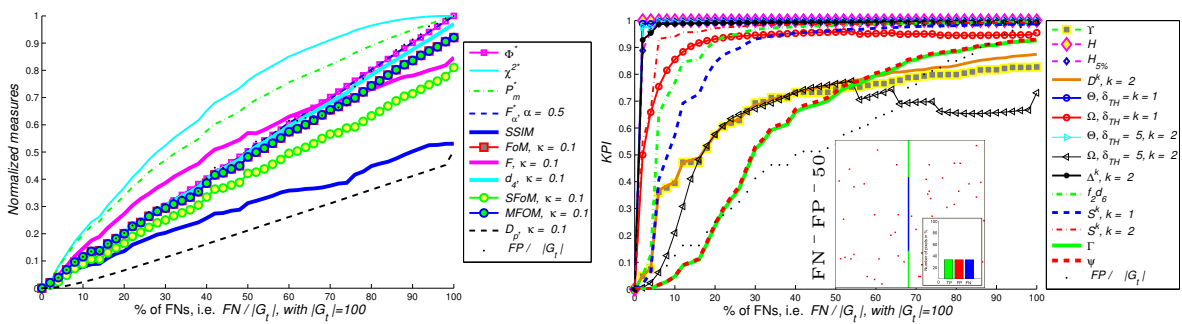

Fig. 6. Dissimilarity measure scores in function of addition of both the FNs and FPs.

time, considering a synthetic edge model (i.e. ground truth) the edge detection evaluation measures are subject to the following studies: addition of false positive points close to the true contour, addition of false negative points (undersegmentation), addition of false positive points (over-segmentation), addition of both false negatives and false positive points, translation of the boundary. Thus, 24 measures and the new proposed method are tested and compared together. The KPI in eq. 4 is computed for the non-normalized algorithms in Table 1.

The first experiment is to create an over-segmentation at a maximal distance of 5 pixels, as illustrated in Fig. 3 (100\% of over-segmentation represents a dilatation of the vertical line with a structural element of size $1 \times 6$, corresponding of a total saturation of the contour, see Fig. 3(d)). Curves presented here show 

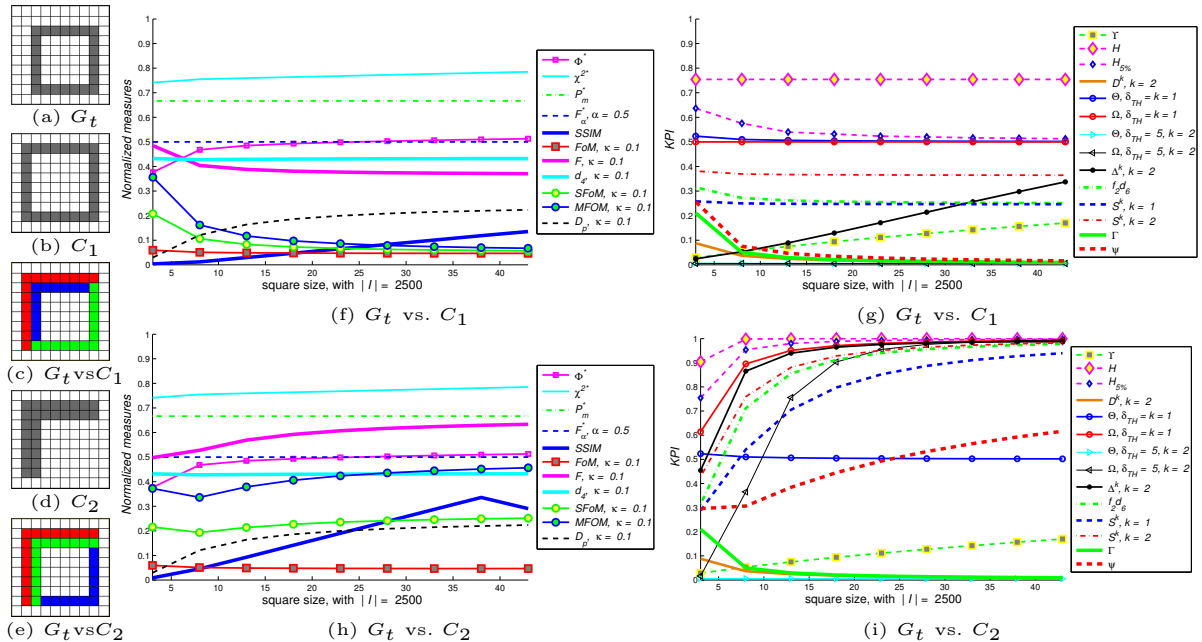

(f) $G_{t}$ vs. $C_{1}$

(g) $G_{t}$ vs. $C_{1}$

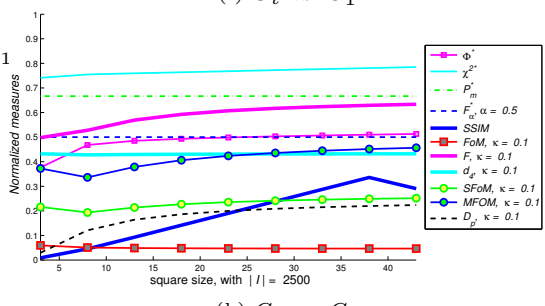

(h) $G_{t}$ vs. $C_{2}$

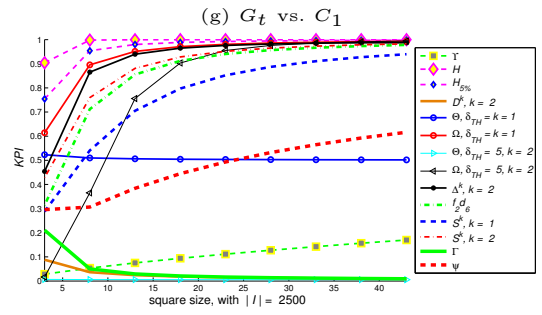

(i) $G_{t}$ vs. $C_{2}$

Fig. 7. Dissimilarity measure scores in function of the size of the original square.

that $F_{\alpha}, d_{4}, F, M F o M, H, H_{5 \%}, \Delta_{k}, D^{k}, f_{2} d_{6}$, and $S^{k}$ are very sensitive to FPs, whereas $S S I M, D_{p}, \Phi$ and $D^{k}$ (which is not homogeneous) do not penalize enough $D_{c}$. $\Omega$ remains constant at 0 because it corresponds to an undersegmentation measure. Moreover, $\Upsilon$ and $F o M$ are not too abrupt, even though they stagnate, like $D^{k}, S F o M, f_{2} d_{6}$, and $S^{k}$. Finally, $\Gamma$ and $\Psi$ are not too abrupt and penalize strongly $D_{c}$ in the presence of many FPs.

The second test is to add random undesirable pixels to $G_{t}$ until $100 \mathrm{FPs}$, as represented in Fig. 4 top left. Globally, the curves in Fig. 4 illustrates that the measures using KPI behave like the previous experiment; only $\Gamma$ and $\Psi$ are not too sensitive to FPs. The normalized evaluation measures increase correctly, but seem stagnant, excepted $\Phi$ and $D_{p}$ which stay close to 0 .

Concerning the addition of FNs, Fig. 5 (left) illustrates that $H, H_{5 \%}$ and $\Omega_{\delta_{T H}=1}$ are very sensitive to the presence of FNs. Also, $D_{p}$ attains only the score of $\frac{1}{2}$. The over-segmentation methods $\Upsilon, D^{k}, \Omega$ and $\Gamma$ remain constant at 0 . On the other hand, the score of the KPI of $\Psi$ attains 0.5 for $50 \%$ of FNs. Afterward, contrary to the addition of FPs or FNs, error measures without distance measures obtains a score of 1 after one pixel of translation and the score of $D_{p}$ stays constant at $\frac{1}{2}$ (Fig. 5 (right)). Only FoM, SFoM, MFoM, the KPI of $\Omega_{\delta_{T H}=5}$, the $K P I$ of $\Gamma$ and the $K P I$ of $\Psi$ behave correctly.

Concerning the line, the last experiment corresponds to an addition of both FPs and FNs. Thus, Fig. 6 shows that the normalized measures, excepted $D_{p}$ and $S S I M$ behave correctly. Concerning other measures, the KPI scores of $\Omega_{\delta_{T H}=5}$, $\Gamma$ and $\Psi$ are not too abrupt for few number or errors and penalize strongly $D_{c}$ in the presence of many FPs and FNs (but $\Omega_{\delta_{T H}=5}$ is not homogeneous). For example, Fig. 6 (bottom right) illustrates the line where both $50 \%$ of the pixels are missing and 50 FPs are added, corresponding to $33 \%$ of TPs. In this precise case, the $K P I$ score of new measure $\Psi$ is close to 0.7 , thus, reflecting the reality. 
Another experiment in Fig. 7, two different shapes are compared to a square $\left(G_{t}\right)$, illustrating the importance to consider both $d_{D_{c}}$ and $d_{G_{t}}$. Furthermore, all the shapes are growing at the same time, keeping the same percentage of FPs and FNs with $G_{t}$. The more $G_{t}$ grows, the more $C_{1}$ is visually closer to $G_{t}$ whereas FNs deviate strongly in the case of $C_{2}$. Despite these tow different evolutions, statistical measures, $F o M, F, d_{4}, D_{p}, \Upsilon$ and $\Gamma$ obtain close the same measurements for $C_{1}$ and $C_{2}$. On the contrary, the $K P I$ of $\Psi$ grows around 0.5 for $C_{2}$, whereas it converges towards 0 for $C_{1}$, since $C_{1}$ becomes visually closer to $G_{t}$ with the enlargement (note that $M F o M$ behaves identically).

To conclude experimental evaluations, Table 2 reports different assessments for five edge detection methods on a real image: Sobel, Canny [10], Steerable Filters of order $1\left(S F_{1}\right)$ [25], Steerable Filters of order $5\left(S F_{5}\right)[26]$ and Half Gaussian Kernels $(H K)$ [6]. Even though the problem of hand-made ground

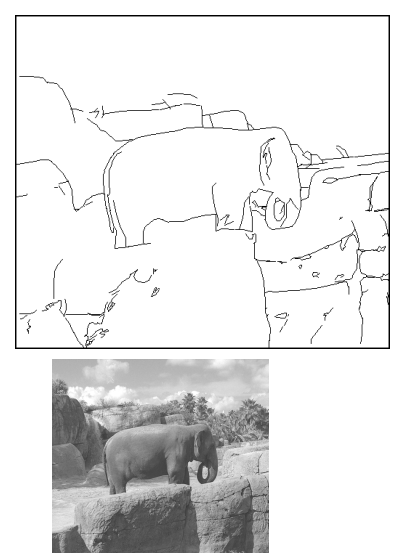

(a) Gt / Original [1]

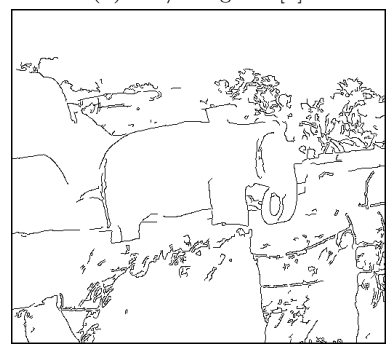

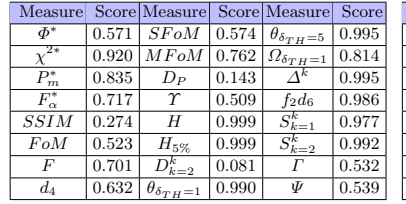

(d) Steerable filters [25]

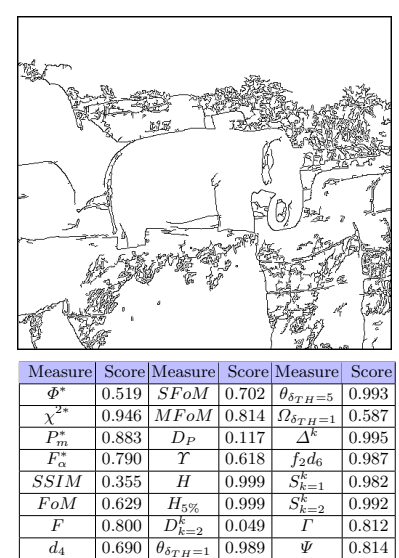

(b) Sobel

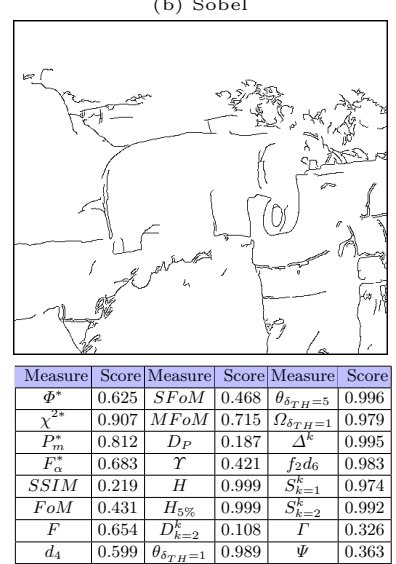

(e) Steerable filters [26]

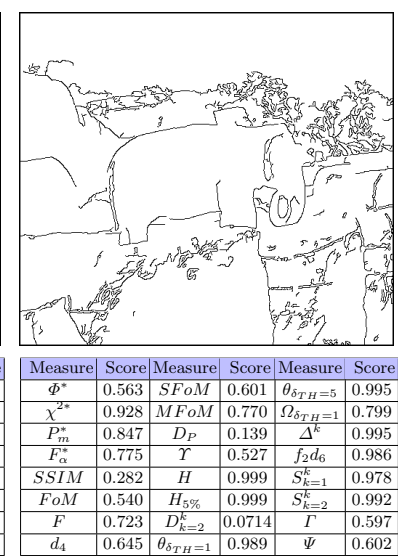

(c) Canny [10]

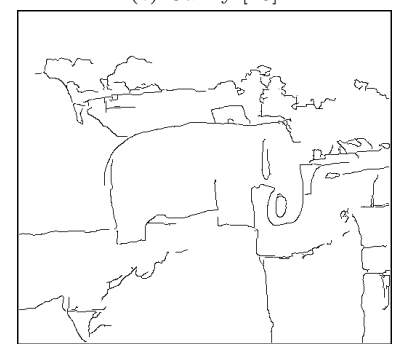

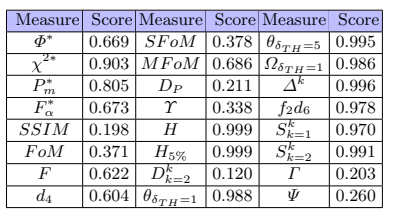

(f) Half Gaussian filters [6]

Table 2. Comparison measures of different edge detections. A score close to 0 indicates a good edge map whereas a score 1 translates a poor segmentation. 
truth on real images is discussed by some researchers [27], only the comparison of $D_{c}$ with a $G_{t}$ is studied here. Compared to $G_{t}$ (Table $2(\mathrm{a})$ ), the well known Sobel edge detector generates more FPs than the other three methods while $S F_{5}$ and $H K$ are less sensitive to noise or texture. Furthermore, $H K$ captures easier straight contours and corners closest to their true positions [6]. So, the measurements in tables of Table 2 must be close to 1 for Sobel and a little less for Canny, but decrease with reasonable error for $H K$ (scores involving $K P I$ for non-normalized algorithms, eq. 4). Thus, $\Gamma$ and $\Psi$ respect this evolution and indicate a good measurement value. FoM, F, $d_{4}, S F o M, M F o M$ and $\Upsilon$ evolve similarly, but the score for the $H K$ remains too elevated. Also, $\Phi^{*}, \chi^{2 *}, P_{m}^{*}, F_{\alpha}^{*}$ and $D_{p}$ do not indicate a significant difference between all the segmentations. Further, other non normalized methods are not adapted to give a score between 0 and 1 using a $K P I$. Eventually, given the segmented images, $\Gamma$ and $\Psi$ indicate a good measurement value. Other results involving other edge images are available on the website: http://hkaljaf .wixsite.com/hasanabdulrahman/edge-detection-and-evaluation.

\section{CONCLUSION AND FUTURE WORKS}

In this paper, several referenced-based boundary detection evaluations are detailed, pointing their advantages and disadvantages through concrete examples of edge images. A new normalized supervised edge map quality measure is proposed, comparing a ground truth contour image, the candidate contour image and their associated spacial nearness. The strategy to normalize the evaluation enables to consider a score close to 0 as a good edge map, whereas a score 1 translates a poor segmentation. Eventually, compared to other edge evaluation assessments, the score of the new evaluation indicates confidently the quality of a segmentation. Next on our work program agenda is to compare different edge detectors with their different parameters and binary image matching.

\section{Acknowledgements}

The authors wish to thank the Iraqi Ministry of Higher Education and Scientific Research for funding and supporting this work.

\section{References}

1. M. D. Heath, S. Sarkar, T. Sanocki, and K. W. Bowyer, "A robust visual method for assessing the relative performance of edge-detection algorithms," IEEE TPAMI, vol. 19, no. 12, pp. 1338-1359, 1997.

2. M-P Dubuisson and Anil K Jain, "A modified hausdorff distance for object matching," in IEEE ICPR, 1994, vol. 1, pp. 566-568.

3. S. Chabrier, H. Laurent, C. Rosenberger, and B. Emile, "Comparative study of contour detection evaluation criteria based on dissimilarity measures," EURASIP J. on Image and Video Processing, vol. 2008, pp. 2, 2008.

4. C. Lopez-Molina, B. De Baets, and H. Bustince, "Quantitative error measures for edge detection," Patt. Rec., vol. 46, no. 4, pp. 1125-1139, 2013. 
5. Z. Wang, A. C. Bovik, H. R. Sheikh, and E. P. Simoncelli, "Image quality assessment: from error visibility to structural similarity," IEEE TIP, vol. 13, no. 4, pp. 600-612, 2004.

6. B. Magnier, P. Montesinos, and D. Diep, "Fast anisotropic edge detection using gamma correction in color images," in IEEE ISPA, 2011, pp. 212-217.

7. B. Magnier, A. Aberkane, P. Borianne, P. Montesinos, and C. Jourdan, "Multiscale crest line extraction based on half gaussian kernels," in IEEE ICASSP, 2014, pp. 5105-5109.

8. K. Bowyer, C. Kranenburg, and S. Dougherty, "Edge detector evaluation using empirical roc curves," in CVIU, 2001, pp. 77-103.

9. D. R. Martin, C. C. Fowlkes, and J. Malik, "Learning to detect natural image boundaries using local brightness, color, and texture cues," IEEE TPAMI, vol. 26, no. 5, pp. 530-549, 2004.

10. J. Canny, "A computational approach to edge detection," IEEE TPAMI, , no. 6, pp. 679-698, 1986.

11. C. Grigorescu, N. Petkov, and M. Westenberg, "Contour detection based on nonclassical receptive field inhibition," IEEE TIP, vol. 12, no. 7, pp. 729-739, 2003.

12. S. Venkatesh and P. L. Rosin, "Dynamic threshold determination by local and global edge evaluation," CVGIP, vol. 57, no. 2, pp. 146-160, 1995.

13. Y. Yitzhaky and E. Peli, "A method for objective edge detection evaluation and detector parameter selection," IEEE TPAMI, vol. 25, no. 8, pp. 1027-1033, 2003.

14. I. E. Abdou and W. K. Pratt, "Quantitative design and evaluation of enhancement/thresholding edge detectors," Proc. of the IEEE, vol. 67, pp. 753-763, 1979.

15. A. J. Pinho and L. B. Almeida, "Edge detection filters based on artificial neural networks," in ICIAP. Springer, 1995, pp. 159-164.

16. A. G. Boaventura and A. Gonzaga, "Method to evaluate the performance of edge detector," 2009.

17. W.A. Yasnoff, W. Galbraith, and J.W. Bacus, "Error measures for objective assessment of scene segmentation algorithms.," Analytical and Quantitative Cytology, vol. 1, no. 2, pp. 107-121, 1978.

18. D.P. Huttenlocher and W.J. Rucklidge, "A multi-resolution technique for comparing images using the hausdorff distance," in IEEE CVPR, 1993, pp. 705-706.

19. T. Peli and D. Malah, "A study of edge detection algorithms," CGIP, vol. 20, no. 1, pp. 1-21, 1982.

20. R. M. Haralick, "Digital step edges from zero crossing of second directional derivatives," IEEE TPAMI, vol. 6, no. 1, pp. 58-68, 1984.

21. C. Odet, B. Belaroussi, and H. Benoit-Cattin, "Scalable discrepancy measures for segmentation evaluation," in IEEE ICIP, 2002, vol. 1, pp. 785-788.

22. A. J. Baddeley, "An error metric for binary images," Robust Computer Vision: Quality of Vision Algorithms, pp. 59-78, 1992.

23. K. Panetta, C. Gao, S. Agaian, and S. Nercessian, "A new reference-based edge map quality measure," IEEE Trans. on Systems Man and Cybernetics: Systems, vol. 46, no. 11, pp. 1505-1517, 2016.

24. B. Magnier, A. Le, and A. Zogo, "A quantitative error measure for the evaluation of roof edge detectors," in IEEE IST, 2016, pp. 429-434.

25. W. T. Freeman and E. H. Adelson, "The design and use of steerable filters," IEEE TPAMI, vol. 13, pp. 891-906, 1991.

26. M. Jacob and M. Unser, "Design of steerable filters for feature detection using canny-like criteria," IEEE TPAMI, vol. 26, no. 8, pp. 1007-1019, 2004.

27. X. Hou, A. Yuille, and C. Koch, "Boundary detection benchmarking: Beyond f-measures," in IEEE CVPR, 2013, pp. 2123-2130. 